\title{
Burning Mouth Syndrome: Patient Profiles, Clinical Symptoms, Affecting Associated Factors, and Treatment Responses
}

\author{
Byung Woo Lim¹, Hae Dong Kim¹, Jin Soon Chang', Ick Soo Choi ${ }^{*}$ \\ ${ }^{1}$ Department of Otorhinolaryngology-Head and Neck Surgery, Ilsan Paik Hospital, Inje University College of Medicine, \\ Ilsanseo-gu, Goyang-si, Korea \\ ${ }^{2}$ Department of Otorhinolaryngology-Head and Neck Surgery, Seoul Paik Hospital, Inje University College of Medicine, \\ Jung-gu, Seoul, Korea \\ Email: *leochoics@gmail.com
}

How to cite this paper: Lim, B.W., Kim, H.D., Chang, J.S. and Choi, I.S. (2018) Burning Mouth Syndrome: Patient Profiles, Clinical Symptoms, Affecting Associated Factors, and Treatment Responses. International Journal of Otolaryngology and Head \& Neck Surgery, 7, 237-248. https://doi.org/10.4236/ijohns.2018.74025

Received: July 9, 2018

Accepted: July 27, 2018

Published: July 30, 2018

Copyright $\odot 2018$ by authors and Scientific Research Publishing Inc. This work is licensed under the Creative Commons Attribution International License (CC BY 4.0).

http://creativecommons.org/licenses/by/4.0/

\begin{abstract}
Background: The clinical characteristics of burning mouth syndrome (BMS) are not fully understood. We investigated the profiles of BMS patients, characteristics associated with BMS, and the available treatment methods and their effects. Methods: Seventy-four BMS patients without oral mucosal lesions were enrolled. Their medical history, medications taken, and symptom scores were investigated via questionnaires. Laboratory investigations of parameters potentially associated with BMS were performed. Regarding treatment, $0.01 \%$ dexamethasone gargle, amitriptyline, and clonazepam were administered individually or in combination, depending on the degree of symptom improvement. Symptoms were scored from 0 - 10 points; these scores were used to evaluate treatment efficacy and patient satisfaction. Results: Mean age of the patients was $63.6 \pm 14.2$ years; mean symptom prevalence period was $15.5 \pm 24.7$ months. BMS was not significantly associated with a history of hypertension, diabetes, or any specific medications. Treatment reduced the symptoms of $85.1 \%$ subjects (63/74). Conclusions: Contrary to that in previous studies, we observed significant improvements in BMS patients following combination treatment with dexamethasone gargle, amitriptyline, and clonazepam.
\end{abstract}

\section{Keywords}

Burning Mouth Syndrome, Dexamethasone, Amitriptyline, Clonazepam

\section{Introduction}

The International Headache Society (IHS) defines burning mouth syndrome 
(BMS) as an "abnormal burning sensation in the mouth that persists for 2 hours or longer per day for 3 months, without a clinically causative lesion" [1]. The International Association for the Study of Pain (IASP) defines it as "chronic pain or discomfort in the mouth without causative lesions and other diseases potentially accounting for the discomfort" [2]. BMS is a relatively rare disease; while reported prevalence rates vary, females aged $\geq 50$ years are reportedly more commonly affected than males [3] [4] [5]. In a 2015 study, the prevalence in the general population was $0.11 \%$ (males: $0.04 \%$, females: $0.17 \%$ ), [3] whereas a 2013 study reported a prevalence rate of $3.7 \%$ in a population aged 20 - 69 years [6]. Despite many reports on the clinical characteristics of BMS, the condition is not yet fully understood. While in most cases its cause is unknown, dental disease has been suggested as a local cause; additionally, mental health issues, chronic adult diseases, and the use of certain drugs may be potential systemic causes [7]. The alleviation of the potential causes is the most commonly prescribed treatment.

Recent studies have reported the use of light therapy [8] and cognitive therapy [9] in BMS treatment. However, there are no well-established treatment method, and treatment effects, if any, are unsatisfactory. While various etiologies have been suggested for BMS, there are no well-established diagnostic, classification, or treatment methods, and most BMS studies have focused solely on the dental area. Thus, investigation of the potentially involved systemic factors is warranted.

The incidence of BMS may be associated with age, chronic disease, and drug use. The discomfort caused by BMS is sometimes so severe that patients cannot manage their daily lives. Primary treatments have thus far been administered in the context of oral medicine, and it has been speculated that most otolaryngologists do not possess the relevant knowledge or experience required for the diagnosis and treatment of BMS [10].

Here, we evaluated patient characteristics and profiles to assess the degree of symptoms and response to our treatment protocol in BMS patients who visited our otolaryngology department during the last eight years.

\section{Methods}

\subsection{Subjects}

We analyzed the records of 74 outpatients who presented at the hospital's Department of Otolaryngology with an oral burning sensation exceeding 3 months in duration as their main complaint between June 2007 and March 2016, and met the study's inclusion criteria. The exclusion criteria were history of head or neck trauma, or oral surgery, and mucosal lesions potentially originating from the mouth. Only patients who reported compliance with the prescribed treatments were included in the analysis. The study design was approved by the appropriate Institutional Review Board. Due to the retrospective nature of the study, the need for informed consent was waived off. 


\subsection{Evaluation Items}

Using a questionnaire, we gathered data on symptom prevalence and duration, affected sites, degrees of discomfort, and accompanying symptoms; patients were allowed to provide multiple answers to the questions. Additionally, information about dental diseases, accompanying systemic diseases, and medication histories was incorporated into the analysis, as well as the presence or absence of menopause and its duration in females.

Blood tests were performed for vitamin B, zinc, iron, folate, thyroid gland function, and erythrocytes, which may be related to BMS [11] [12]. Cortisol and adenocorticotropic hormone (ACTH) levels were also measured, to evaluate the systemic effects of long-term use of dexamethasone gargle.

Saliva tests using citric acid were conducted to examine xerostomia, because many previous studies have suggested there is a relationship between xerostomia and BMS [12] [13] [14].

Data were organized in the Excel spreadsheets (Microsoft Excel 2017 ${ }^{\circledR}$, Microsoft Corporation, and U.S.) and coded appropriately for statistical analysis.

\subsection{Treatment Methods and Determination of Treatment Responses}

Patients with possible causative factors were treated by correcting nutritional or hormonal factors, and changing or suspending relative drugs for 4 weeks. If symptoms completely disappeared after changing drugs, the original drug treatments were terminated; otherwise, the original drug treatments were continued. For BMS treatment, dexamethasone gargle, amitriptyline, or clonazepam were selected based on the prior experience of the authors and a review of the literature [11] [15] [16] [17]. The treatment protocol is shown in Figure 1. Drugs were consecutively added to the regimen based on patient satisfaction regarding the improvement of symptoms, and patients visited the outpatient department every 2 weeks to rate the improvement or aggravation of their symptoms

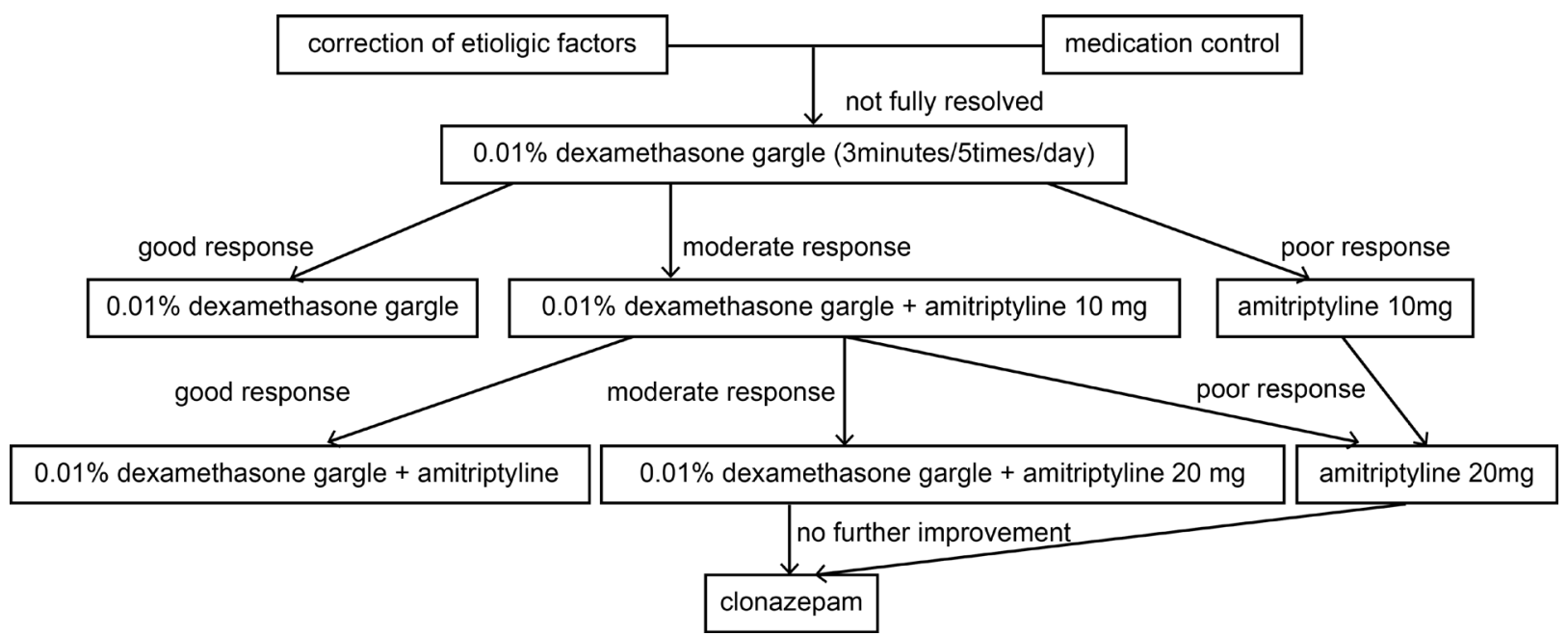

Figure 1. Flow diagram of the treatment protocol. 
via a symptom score, where 0 denoted "no discomfort" and 10 denoted "extreme discomfort or pain". The satisfaction of patients with symptom improvement was also considered when prescribing drug treatments; this was measured as satisfied, somewhat satisfied, and dissatisfied. After summing these two measures, the overall response was classified as follows: A-B points, good response (Marked improvement); C-D points, moderate response (Slight improvement); E-F points, poor response (No change). Although some patients had a history of psychiatric medication, no patient in the group that used either amitriptyline or clonazepam as their final treatment had used the same drug previously.

\section{Results}

\subsection{Patients Profile and Clinical Symptoms}

We included 74 patients, 57 female (77\%) and 17 male (23\%), and their mean age was $63.55 \pm 14.17$ years (range 23 to 89 years). Of the 57 female patients, 46 (81\%) were in menopause. The mean symptom prevalence period was $15.48 \pm$ 24.73 months with a range of 3 to 120 months, and the age group with the highest incidence was patients in their 60s for both males and females (Figure 2). The most commonly reported symptom was burning (36 patients, $49 \%$ ), and the most common sites of discomfort were the tongue (59 patients, $80 \%$ ) and the entire mouth (16 patients, 22\%). Thirty-five patients (47\%) reported the persistence of symptoms throughout the day. Regarding associated symptoms, the

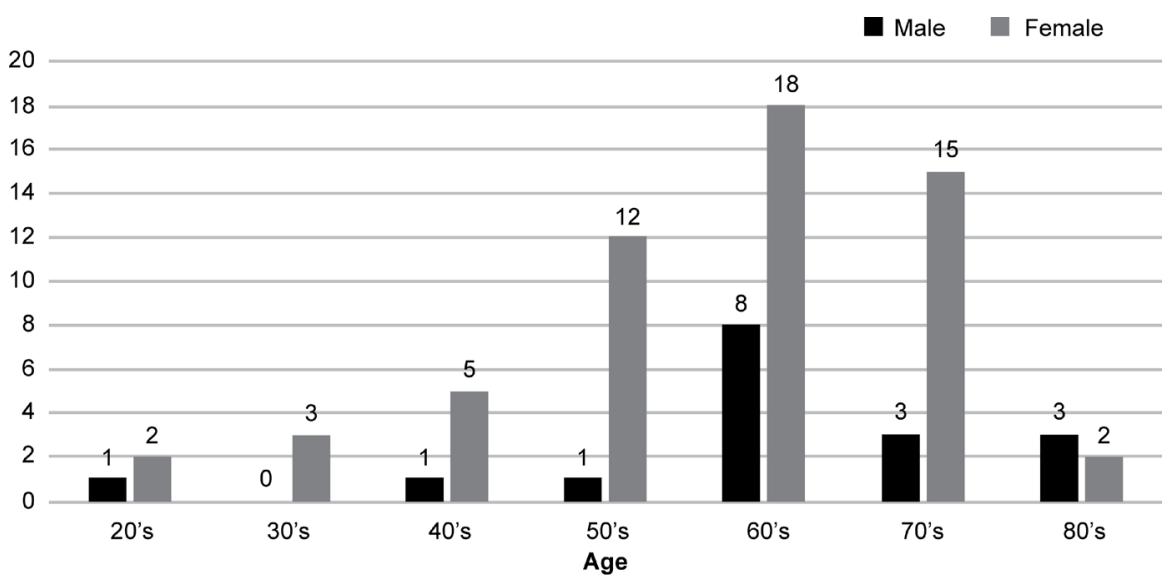

(a)

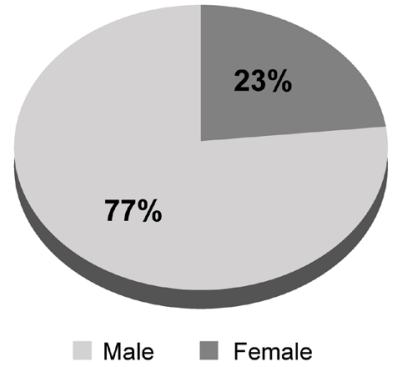

(b)

Figure 2. (a) Patient distribution according to age at onset, and sex (b) Sex ratio of BMS patients. 
most common was dryness of the mouth (42 patients, 57\%; 14 exhibited abnormal findings in both non-stimulated and stimulated salivary tests), followed by tingling in the mouth (36 patients, 49\%). The symptoms and sites of discomfort are summarized in Table 1.

\subsection{Associated Factors}

Of the 74 patients, $50(68 \%)$ patients had more than one possible factor related with BMS. Thirty-eight patients had a medication history (including eight multifactorial), with psychiatric medication being the most frequent, 28 had a nutritional deficiency or hormonal abnormality (including eight multifactorial), with iron deficiency being the most common, and eight had both factors (Table 2).

\subsection{Treatment Responses}

Because no patients with associated factors were satisfied with the correction of etiologic factors or medication control, all 74 patients needed medication. After treatment completion, we analyzed the outcomes in all patients, and in sub-groups according to treatment drug.

All patients. Sixty-three patients ( $85 \%$ of the total) reported satisfaction with

Table 1. Distribution of patients according to the BMS symptoms reported, the locations of these symptoms, and associated symptoms.

\begin{tabular}{|c|c|c|}
\hline Symptoms reported & $n$ & Percentage \\
\hline Burning & 36 & $49 \%$ \\
\hline Stinging & 27 & $36 \%$ \\
\hline Soreness & 8 & $11 \%$ \\
\hline Pricking & 22 & $30 \%$ \\
\hline Smarting & 22 & $30 \%$ \\
\hline Fiery & 16 & $22 \%$ \\
\hline \multicolumn{3}{|l|}{ Locations } \\
\hline Tongue & 59 & $80 \%$ \\
\hline Entire mouth & 16 & $22 \%$ \\
\hline Gums & 5 & $7 \%$ \\
\hline Palate & 5 & $7 \%$ \\
\hline Lips & 9 & $12 \%$ \\
\hline Mouth floor & 5 & $7 \%$ \\
\hline \multicolumn{3}{|l|}{ Associated symptoms } \\
\hline Xerostomia & 42 & $57 \%$ \\
\hline Dysgeusia & 23 & $31 \%$ \\
\hline Cacogeusia & 12 & $16 \%$ \\
\hline Tingling & 36 & $49 \%$ \\
\hline None & 3 & $4 \%$ \\
\hline
\end{tabular}


Table 2. Distribution of patients according to association of related factors.

\begin{tabular}{|c|c|}
\hline None & 24 \\
\hline Medication history & 38 \\
\hline Psychiatric (PSY) & 8 \\
\hline Hypertension(HBP) & 6 \\
\hline Diabetes(DM) & 0 \\
\hline Two more & 16 \\
\hline $\mathrm{PSY}+\mathrm{HBP}$ & 7 \\
\hline $\mathrm{HBP}+\mathrm{DM}$ & 4 \\
\hline $\mathrm{PSY}+\mathrm{DM}$ & 3 \\
\hline All three & 6 \\
\hline Nutritional deficiency or hormonal abnormality & 12 \\
\hline Iron & 6 \\
\hline Zinc & 2 \\
\hline Vit B12 & 0 \\
\hline Hypothyroidism & 0 \\
\hline Hyperthyroidism & 2 \\
\hline Two more; & 2 \\
\hline Vit B12+ Zinc & 1 \\
\hline Iron + Zinc + hypothyroidism & 1 \\
\hline Both & 8 \\
\hline
\end{tabular}

the treatment and exhibited improvements in symptom scores. Twenty-three (31\%) exhibited symptom improvement via dexamethasone gargle alone, 33 (45\%) exhibited improvement either via dexamethasone gargle and amitriptyline (14 points), or only amitriptyline (19 points) as the final drug, and 7 (9\%) exhibited improvement after using clonazepam. None of the 37 patients who underwent long-term steroid gargle treatment exhibited abnormal values in cortisol or ACTH tests. Eleven (15\%) did not experience symptom improvement even after treatment with all of the drugs. The treatment outcomes according to association factor are presented in Figure 3. An analysis of the patients grouped by the final drug/s used and the association of "possible" causative factors is presented in Figure 4. While the mean symptom score at the first outpatient visit was $7.22 \pm$ 2.12 points, at the second outpatient visit after 2 weeks it was $5.11 \pm 2.62$ and at the final visit it was $3.46 \pm 2.66$, indicating a continuous improvement of symptoms.

Analysis of effects grouped by final drug/s used. The patients were divided into three groups depending on the final drug/s used, as follows: group D was treated with dexamethasone gargle alone, group $\mathrm{D}+\mathrm{A} / \mathrm{A}$ was treated with either dexamethasone gargle and amitriptyline, or amitriptyline alone, and group $\mathrm{C}$ was treated with clonazepam. 


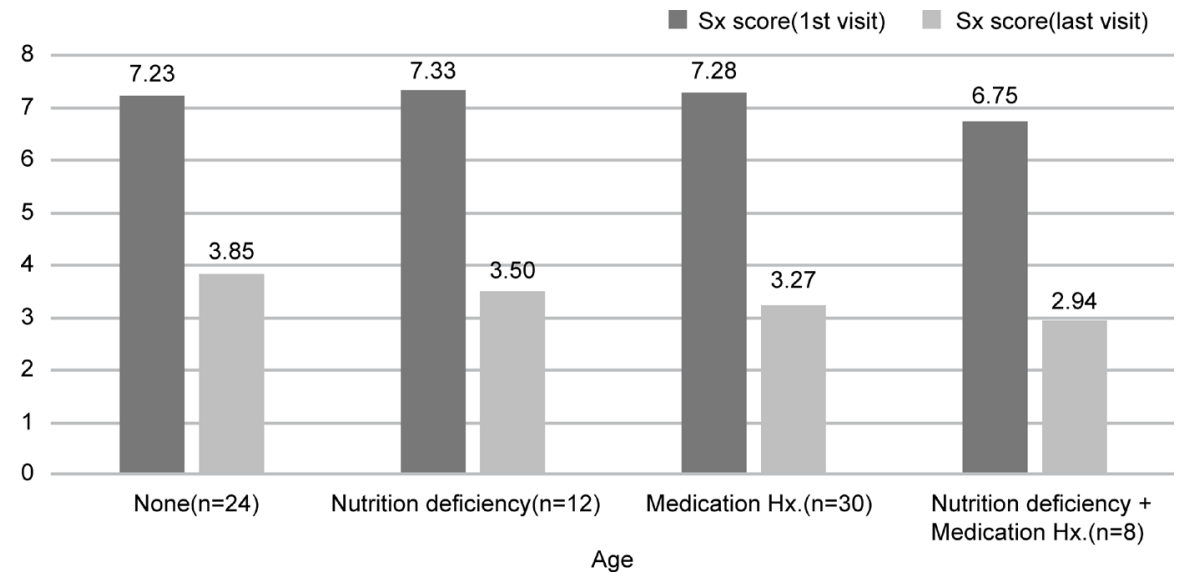

Figure 3. First and last visit symptom scores according to association of possible causative factors. Hx: history; Sx: symptom.

(a)

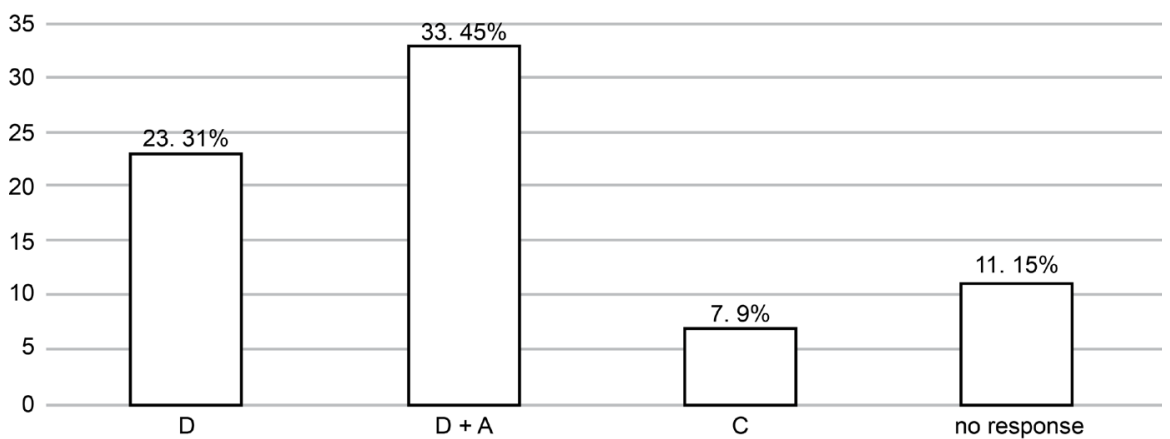

(b)

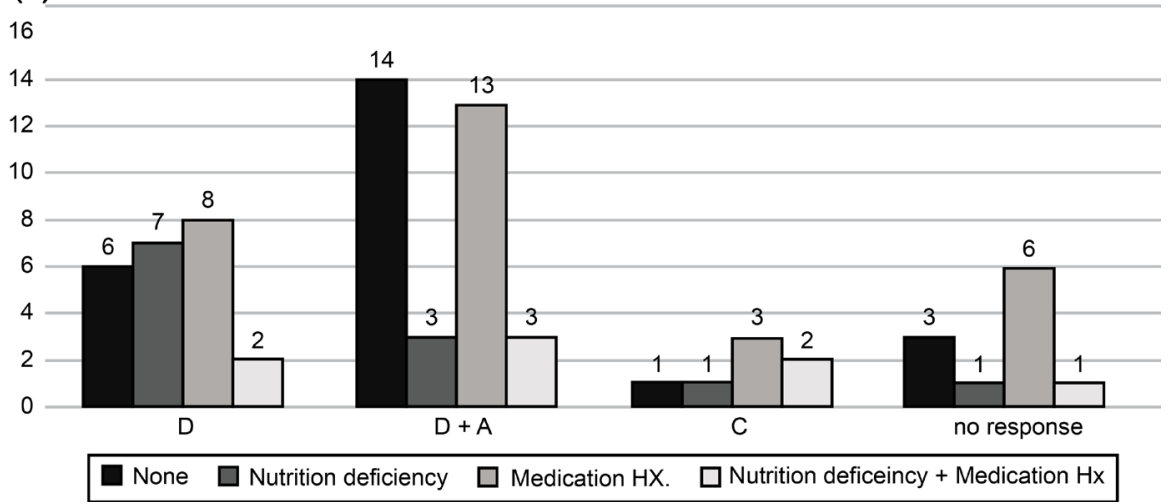

Figure 4. (a) The number of patients in each treatment group and the composition of the groups; (b) The distribution of the treatment groups according to the symptom classification. D: dexamethasone gargle alone; D + A/A: dexamethasone gargle and amitriptyline, or amitriptyline alone; C: clonazepam; Hx: history.

Group D included 23 patients (31\%). Their mean symptom scores were $6.74 \pm$ $2.26,3.90 \pm 2.52$, and $2.94 \pm 2.49$ at the first, second, and final outpatient visit, respectively. Group D+A/A included 33 patients (45\%), and their mean symptom score was $7.29 \pm 1.99$ at the first outpatient visit. At the second visit, after using dexamethasone gargle only, it was $5.46 \pm 2.59$. While this does represent an improvement, the satisfaction of these patients was not high, so amitriptyline 
was prescribed. Their final mean symptom score was $3.65 \pm 2.83$. Group C included 7 patients (9\%). Their mean symptom scores were $8.13 \pm 2.30,6.63 \pm$ 1.71 , and $3.56 \pm 1.92$ at the first, second, and final outpatient visit, respectively (Table 3).

\section{Discussion}

The term "BMS" was first used in the 1960s, and used to refer to any burning sensation in the mouth. Now, it refers to not only a burning sensation in the mouth, but also other accompanying symptoms; thus the term "syndrome" is used.

Since many otolaryngologists rarely encounter BMS patients and thus may have difficulty accurately diagnosing it, its prevalence has been variably reported, ranging from $0.11 \%$ to $17 \%$ [3] [12] [18]. This is likely due to the absence of clear diagnostic criteria. In fact, different BMS studies have applied different criteria. Gary et al. [19] attempted to collate and summarize the opinions of 15 specialist North American dentists about the definition and diagnostic criteria for BMS, and their hypotheses regarding its etiology. Despite efforts such as this, clearly established criteria for the diagnosis of BMS do not exist yet.

While the primary cause of BMS is unclear, one previous study classified the disease as primary or secondary depending on the presence or absence of potential causes, and reported that various factors could be involved in secondary BMS [4]. Two hypotheses have been suggested regarding the etiology of primary BMS: its onset may be due to a neuropathic condition, 5 or due to an underlying psychiatric condition [12]. In contrast, secondary BMS is thought to be caused by interactions among local, systemic, and psychological factors [12]. Suspected local factors include physical or chemical stimuli in the mouth, oral mucosal lesions, dryness of the mouth, and bacterial, fungal, or viral infections [12]. Suspected systemic factors include deficiencies in B group vitamins, folate, iron, and zinc, which are thought to be associated with the enzymes responsible for functional changes in epithelium, resulting in BMS symptoms [12]. In addition, BMS may be affected by diseases associated with reduced salivary gland secretion such as diabetes and thyroid disease, and endocrine changes such as hormonal changes associated with menopause, Sjögren's syndrome, and sicca syndrome, as well as by drugs and gastroesophageal reflux disease [4] [12] [20]. Regarding psychological factors, depression, anxiety, obsessive disorders, and somatization disorder may be associated with BMS [21]. A better understanding of these

Table 3. Mean symptom scores of each treatment group at the first, second, final visit.

\begin{tabular}{cccc}
\hline & $1^{\text {st }}$ Visit & 2 week & Final \\
\hline D & $6.74 \pm 2.26$ & $3.90 \pm 2.52$ & $2.94 \pm 2.49$ \\
D + A /A & $7.29 \pm 1.99$ & $5.46 \pm 2.59$ & $3.65 \pm 2.83$ \\
C & $8.13 \pm 2.30$ & $6.63 \pm 1.71$ & $3.56 \pm 1.92$ \\
All & $7.22 \pm 2.18$ & $5.11 \pm 2.27$ & $3.46 \pm 2.41$ \\
\hline
\end{tabular}


potential causative factors is required for the diagnosis and treatment, as well as prevention of BMS in patients with these conditions. Approximately $68 \%$ of the patients in the present study were found to have at least one of the previously reported potentially causative factors, thus attempts were made to treat these conditions in those patients, for example via the supplementation of nutrients or hormones to restore normal levels, or suspension of relevant drug regimens, followed by assessment of treatment responses. If treatment for a potentially contributing factor improves symptoms, that factor can be considered a potential cause. However, in the present study only one patient who exhibited vitamin deficiency responded to supplementation (and only partially); no cases in which potentially involved drugs were suspended or altered showed significant improvement, and hormone supplementation was similarly ineffective. Thus, it may be that these potentially causative factors were present by chance. Alternatively, because the final assessment was only 4 weeks after the initiation of treatment, the duration may not have been long enough to induce a significant improvement in symptoms. It is also possible that irreversible changes in the nerve endings of the oral mucosa were present. In the current study, most cases showed no improvement of symptoms even after the correction of potentially causative factors, so patients were not classified in terms of primary or secondary BMS. Instead, they were grouped based on the presence or absence of a potentially causative factor. After the undergoing treatment, both groups showed comparable treatment effects $(84.0 \%$ for those with potentially causative factors and $87.5 \%$ for those without potentially causative factors; Figure 4 ). To date, studies on the potentially causative factors described above have failed to establish them as definitively causative, and no reliable data confirming that the treatment of these potential causes has a beneficial effect on BMS have been reported. On the contrary, some prior studies have suggested that these factors may not be associated with BMS [11] [22] [23]. Consistent with this suggestion, the present study failed to identify a reduction in saliva secretion in BMS patients. Therefore, the factors potentially associated with BMS should be studied further, to clearly identify causative factors.

BMS can cause substantial discomfort in patients, and the most commonly reported manifestation of it is a burning sensation at the tip of the tongue [11]. In the present study, approximately $80 \%$ of BMS patients reported this symptom. BMS is also known to be accompanied by other symptoms in some cases, including alterations in the sense of taste, olfactory abnormalities, and dryness of the mouth. Of these, dryness of the mouth is one of the most common accompanying symptoms reported by patients. In the present study, 57\% of BMS patients complained of dryness of the mouth. However, saliva tests investigating xerostomia revealed that only $30 \%$ of patients had reduced salivary gland function, while $57 \%$ of patients complained of dryness of the mouth, suggesting that dryness of the mouth may be only weakly correlated with salivary gland function. 
Despite the investigation of various drugs and procedures for the treatment of BMS, most treatments are applied to control the symptoms rather than to treat the disease itself, because the etiology of BMS is not fully understood. In the present study, dexamethasone gargle was applied based on the hypothesis that the potent anti-inflammatory activity of the steroid may be effective in combating the hypersensitivity caused by the inflammatory reactions of nerve endings. Dexamethasone gargle is one of the most non-invasive treatment options, and Choung et al. [11] have reported improvements in approximately $50 \%$ of cases following such treatment, thus it was utilized as the initial treatment in the present study. The dexamethasone formulation used by Choung et al. [11] was a $0.006 \%$ gargle liquid consisting of $300 \mathrm{~mL}$ physiological saline and $20 \mathrm{mg}$ dexamethasone. In contrast, the present study used a $0.01 \%$ gargle liquid, which was expected to result in a better outcome because this concentration has been successfully used for the treatment of oral lichen planus [24]. Only $31 \%$ of patients reported satisfactory results via treatment with dexamethasone gargle alone in the present study, so it could be argued that a $0.01 \%$ concentration is less effective than a $0.006 \%$ concentration. However, as treatment effects were determined based on self-reported patient satisfaction, it may be unreasonable to compare the effects of the two concentrations directly. In addition, the tricyclic anti-depressant amitriptyline is known to be effective for treating chronic neuropathic pain, via inhibition of the reuptake of neurotransmitters [16].

The effects of clonazepam have been reported in many studies [25]. As its use for an extended period of time is reportedly associated with adverse effects including drug dependency, resistance, and withdrawal symptoms, in the present study it was used as the final drug treatment option [17].

Various agents, including clonazepam mouth wash, capsaicin, and $\alpha$-lipoic acid, are reportedly beneficial in BMS patients [26] [27] [28]. The effectiveness of cognitive therapy [29] and light therapy [8] for BMS also have been reported. However, there is as yet no standard treatment regimen for BMS. The treatment method described in the present study improved symptoms in approximately $85 \%$ of patients, in which pain decreased by an average of approximately $52 \%$. Based on the observed effects of initial steroid gargle treatment for 2 weeks, it may be possible to predict the prognosis of BMS patients with greater accuracy.

BMS, a disease of unknown etiology, has been actively studied. Numerous studies have investigated potential treatments, and dexamethasone gargle, amitriptyline, and clonazepam effectively improved symptoms in many patients in the present study. However, only a small number of patients reported the complete alleviation of discomfort. The etiology of BMS remains uncharacterized, and thus requires further study.

\section{Conflicts of Interest}

The authors declare no conflicts of interest regarding the publication of this paper. 


\section{References}

[1] Crow, H.C. and Gonzalez, Y. (2013) Burning Mouth Syndrome. Oral and Maxillofacial Surgery Clinics of North America, 25, 67-76. https://doi.org/10.1016/j.coms.2012.11.001

[2] Merskey, H. and Bogduk, N. (1994) Glossodynia and Sore Mouth. Classification of Chronic Pain. IASP Press, Seattle.

[3] Kohorst, J.J., Bruce, A.J., Torgerson, R.R., Schenck, L.A. and Davis, M.D. (2015) The Prevalence of Burning Mouth Syndrome: A Population-Based Study. British Journal of Dermatology, 172, 1654-1656. https://doi.org/10.1111/bjd.13613

[4] Nasri-Heir, C., Zagury, J.G., Thomas, D. and Ananthan, S. (2015) Burning Mouth Syndrome: Current Concepts. The Journal of Indian Prosthodontic Society, 15, 300-307. https://doi.org/10.4103/0972-4052.171823

[5] Chang, J.W. and Kim, C.H. (2013) Burning Mouth Syndrome. Korean Journal of Otorhinolaryngology - Head and Neck Surgery, 56, 550-556. https://doi.org/10.3342/kjorl-hns.2013.56.9.550

[6] Bergdahl, M. and Bergdahl, J. (1999) Burning Mouth Syndrome: Prevalence and Associated Factors. Journal of Oral Pathology \& Medicine, 28, 350-354. https://doi.org/10.1111/j.1600-0714.1999.tb02052.x

[7] Coculescu, E.C., Manole, G., Coculescu, B.I. and Purcărea, V.L. (2015) Burning Mouth Syndrome: Controversial Place as a Symptom of Oro-Dental Pathology. Journal of Medicine and Life, 8, 34-37.

[8] dos Santos, L.D., Andrade, S.C., Nogueira, G.E., Leão, J.C. and de Freitas, P.M. (2015) Phototherapy on the Treatment of Burning Mouth Syndrome: A Prospective Analysis of 20 Cases. Photochemistry and Photobiology, 91, 1231-1236.

https://doi.org/10.1111/php.12490

[9] Komiyama, O., Nishimura, H., Makiyama, Y., et al. (2013) Group Cognitive-Behavioural Intervention for Patients with Burning Mouth Syndrome. Journal of Oral Science, 55, 17-22. https://doi.org/10.2334/josnusd.55.17

[10] Ducasse, D., Courtet, P. and Olie, E. (2013) Burning Mouth Syndrome: Current Clinical, Physiopathologic, and Therapeutic Data. Regional Anesthesia and Pain Medicine, 38, 380-390. https://doi.org/10.1097/AAP.0b013e3182a1f0db

[11] Choung, Y.H., Cho, M.J., Kim, C.H., Lee, J. and Kang, S.O. (2004) Preliminary Results of Steroid Gargle Treatment and Clinical Characteristics of Patients with Burning Mouth Syndrome. Korean Journal of Otorhinolaryngology, 47, 569-574.

[12] Coculescu, E.C., Tovaru, S. and Coculescu, B.I. (2014) Epidemiological and Etiological Aspects of Burning Mouth Syndrome. Journal of Medicine and Life, 7, 305-309.

[13] Hershkovich, O. and Nagler, R.M. (2004) Biochemical Analysis of Saliva and Taste Acuity Evaluation in Patients with Burning Mouth Syndrome, Xerostomia and/or Gustatory Disturbances. Archives of Oral Biology, 49, 515-522. https://doi.org/10.1016/j.archoralbio.2004.01.012

[14] López-Jornet, P., Camacho-Alonso, F. and Bermejo-Fenoll, A. (2006) A Simple Test for Salivary Gland Hypofunction Using Oral Schirmer's Test. Journal of Oral Pathology \& Medicine, 35, 244-248. https://doi.org/10.1111/j.1600-0714.2006.00411.x

[15] Miziara, I., Chagury, A., Vargas, C., Freitas, L. and Mahmoud, A. (2015) Therapeutic Options in Idiopathic Burning Mouth Syndrome: Literature Review. International Archives of Otorhinolaryngology, 19, 86-89.

https://doi.org/10.1055/s-0034-1378138 
[16] Sharav, Y., Singer, E., Schmidt, E., Dionne, R.A. and Dubner, R. (1987) The Analgesic Effect of Amitriptyline on Chronic Facial Pain. Pain, 31, 199-209. https://doi.org/10.1016/0304-3959(87)90036-4

[17] Cui, Y., Xu, H., Chen, F.M., et al. (2016) Efficacy Evaluation of Clonazepam for Symptom Remission in Burning Mouth Syndrome: A Meta-Analysis. Oral Disease, 22, 503-511. https://doi.org/10.1111/odi.12422

[18] Zakrzewska, J.M., Forssell, H. and Glenny, A.M. (2005) Interventions for the Treatment of Burning Mouth Syndrome. The Cochrane Database of Systematic Reviews, 25, CD002779. https://doi.org/10.1002/14651858.CD002779.pub2

[19] Klasser, G.D., Pinto, A., Czyscon, J.M., Cramer, C.K. and Epstein, J. (2013) Defining and Diagnosing Burning Mouth Syndrome: Perceptions of Directors of North American Postgraduate Oral Medicine and Orofacial Pain Programs. JADA, 144, 1135-1142. https://doi.org/10.14219/jada.archive.2013.0031

[20] das Neves de Araújo Lima, E., Barbosa, N.G., Dos Santos, A.C., et al. (2016) Comparative Analysis of Psychological Hormonal and Genetic Factors between Burning Mouth Syndrome and Secondary Oral Burning. Pain Medicine, 17, 1602-1611. https://doi.org/10.1093/pm/pnv087

[21] Abetz, L.M. and Savage, N.W. (2009) Burning Mouth Syndrome and Psychological Disorders. Australian Dental Journal, 54, 84-93. https://doi.org/10.1111/j.1834-7819.2009.01099.x

[22] Ship, J.A., Grushka, M., Lipton, J.A., Mott, A.E., Sessle, B.J. and Dionne, R.A. (1995) Burning Mouth Syndrome: An Update. The Journal of the American Dental Association, 126, 842-853. https://doi.org/10.14219/jada.archive.1995.0305

[23] Basker, R.M., Sturdee, D.W. and Davenport, J.C. (1978) Patients with Burning Mouths. A Clinical Investigation of Causative Factors, Including the Climacteric and Diabetes. British Dental Journal, 145, 9-16. https://doi.org/10.1038/sj.bdj.4804107

[24] Edwards, P.C. and Kelsch, R. (2002) Oral Lichen Planus: Clinical Presentation and Management. Journal of the Canadian Dental Association, 68, 494-499.

[25] Heckmann, S.M., Kirchner, E., Grushka, M., Wichmann, M.G. and Hummel, T. (2012) A Double-Blind Study on Clonazepam in Patients with Burning Mouth Syndrome. Laryngoscope, 122, 813-816. https://doi.org/10.1002/lary.22490

[26] De Castro, L.A. and Ribeiro-Rotta, R.F. (2014) The Effect of Clonazepam Mouthwash on the Symptomatology of Burning Mouth Syndrome: An Open Pilot Study. Pain Medicine, 15, 2164-2165. https://doi.org/10.1111/pme.12552

[27] Femiano, F., Gombos, F. and Scully, C. (2004) Burning Mouth Syndrome: The Efficacy of Lipoic Acid on Subgroups. Journal of the European Academy of Dermatology and Venereology, 18, 676-678. https://doi.org/10.1111/j.1468-3083.2004.01049.x

[28] De Moraes, M., do Amaral Bezerra, B.A., da Rocha Neto, P.C., de Oliveira Soares, A.C., Pinto, L.P. and de Lisboa Lopes Costa, A. (2012) Randomized Trials for the Treatment of Burning Mouth Syndrome: An Evidence-Based Review of the Literature. Journal of Oral Pathology \& Medicine, 41, 281-287.

https://doi.org/10.1111/j.1600-0714.2011.01100.x

[29] Komiyama, O., Obara, R., Uchida, T., et al. (2012) Pain Intensity and Psychosocial Characteristics of Patients with Burning Mouth Syndrome and Trigeminal Neuralgia. Journal of Oral Science, 54, 321-327. https://doi.org/10.2334/josnusd.54.321 